\title{
Contours, perspectives et tensions des « humanités numériques »
}

Le thème des humanités numériques (HN) est fortement mobilisé dans la récente production scientifique, et devient un élément prépondérant dans les différents projets qui structurent le paysage académique (Longhi 2017) ${ }^{1}$. Si les humanités numériques « représentent bien plus qu'un mouvement de mode passager et superficiel, quoi qu'en disent les mauvaises langues » mais sont « un véritable mouvement de fond appelé à redéfinir l'ensemble des champs de la recherche en sciences humaines et sociales » (Dacos et Mounier, 2014 : 6), elles posent de nombreuses questions quant à la définition de leur périmètre, en relation, à la fois, avec ce que recouvrent les termes « humanités » et « numériques».

\section{Introduction}

Dans cette qualification, et ce syntagme « humanités numériques », il y a un potentiel discursif, dû non seulement à la polysémie des termes « humanités » et « numériques », mais aussi en lien avec le statut de cette qualification (qualification? Catégorisation?). Il existe bien sûr de nombreux travaux qui ont interrogé cette définition même. La page Wikipedia « Humanités numériques » les définit ainsi :

Les humanités numériques (traduction française de digital humanities, abrégées « DH », voire humanités digitales) sont un domaine de recherche,

1. Je remercie Jean-Guy Meunier pour les nombreux échanges que nous avons eus à propos des Humanités Numériques. 
d'enseignement et d'ingénierie au croisement de l'informatique et des arts, lettres, sciences humaines et sciences sociales.

Elles se caractérisent par des méthodes et des pratiques liées à l'utilisation et au développement d'outils numériques en sciences humaines et sociales (les humanities computing, ou " humanités computationnelles »), en ligne et hors ligne, ainsi que par la volonté de prendre en compte les nouveaux contenus et médias numériques, au même titre que des objets d'étude plus traditionnels (les digital studies, ou " études numériques »).

Les humanités numériques s'enracinent souvent d'une façon explicite dans un mouvement en faveur de la diffusion, du partage et de la valorisation du savoir.

Ici, les HN sont définies comme « un domaine de recherche, d'enseignement et d'ingénierie ", avec un double rapport entre humanités et numérique: les outils numériques pour les SHS, et les contenus numériques pour les études en SHS.

Dans le « Manifeste des Digital Humanities » (http://tcp.hypotheses. org/ 318), une définition est proposée (cette définition est liée et proposée par les " acteurs ou observateurs des digital humanities (bumanités numériques) nous sommes réunis à Paris lors du THAT Camp des 18 et 19 mai 2010»):

1. Le tournant numérique pris par la société modifie et interroge les conditions de production et de diffusion des savoirs.

2. Pour nous, les digital humanities concernent l'ensemble des Sciences humaines et sociales, des Arts et des Lettres. Les digital humanities ne font pas table rase du passé. Elles s'appuient, au contraire, sur l'ensemble des paradigmes, savoir-faire et connaissances propres à ces disciplines, tout en mobilisant les outils et les perspectives singulières du champ du numérique.

3. Les digital humanities désignent une transdiscipline, porteuse des méthodes, des dispositifs et des perspectives heuristiques liés au numérique dans le domaine des Sciences humaines et sociales.

Ici, c'est plus explicitement les sciences humaines qui sont mises au centre (voir le point 3). Elles sont décrites comme croisement de disciplines, ou transdiscipline, en considérant la manière dont les différents paradigmes des SHS se trouvent impactés par le numérique. Le numérique semble à la fois être envisagé dans sa dimension informatique (outils, développements, etc.) et dans ses pratiques. Une difficulté qui apparaît dès lors que l'on essaye de caractériser, cartographier, ou pratiquer, les humanités numériques. Une difficulté tient selon nous notamment au rapport à l'informatique/numérique, puisque la thématisation des humanités numériques semble passer prioritairement par le prisme des humanités. Cette réflexion est donc propice à observer ce que la revue Sens-Dessous appelle le « dessous » qui « définit la complexité des expériences intellectuelles ou existentielles vécues, régulièrement évacuée par les discours consensuels ou la recherche d'une vérité d'un seul tenant ».

À travers l'interrogation des différentes acceptions des « humanités » et du " numérique ", et la mise en perspective de différentes manières de concevoir ou pratiquer les humanités numériques, nous montrerons dans cet article que

- d'un côté, les humanités ne peuvent continuer à prendre l'informatique simplement comme un réservoir d'outils sans savoir la manière dont ils sont 
concrètement conçus (la « boîte noire »), ou pourquoi et comment ils sont pertinents pour leur recherche: si tel est le cas, elles y perdront leur signature propre;

- d'un autre côté, les informaticiens ne peuvent continuer à appliquer aveuglément des outils qui fonctionnent correctement ailleurs et qui sont déclarés applicables dans les humanités: si tel est le cas alors les humanités perdront en qualité de résultats. Il s'agit donc de penser les humanités numériques comme une co-construction des objets, savoirs et outils, avec une prise en compte raisonnée et réciproque des enjeux et attendus, des humanités et de l'informatique.

\section{Humanités, sciences humaines, sciences hu-} maines et sociales

Edmond Ortigues, dans son article « Sciences Humaines » de l'Encyclopædia Universalis, explique l'institutionnalisation des sciences humaines :

Par un décret du 23 juillet 1958 (publié au Journal officiel du 27 juillet 1958), on transforma les facultés des lettres en facultés des lettres et sciences humaines, dans le dessein d'y promouvoir l'enseignement d'une partie des sciences sociales (la psychologie et la sociologie), au voisinage des humanités littéraires. Dans ce sens académique, passé dans l'usage, l'expression « sciences humaines » est une locution idiomatique typiquement française (l'anglais l'emploie parfois dans des contextes assez lâches, mais dit plus couramment social sciences)

Mais les «sciences humaines », ou les « lettres et sciences humaines ", ou encore les « lettres, sciences humaines et sociales " ne se confondent pas avec les humanités, même si elles peuvent être considérées comme une transposition institutionnelle de ce que ces dernières recouvrent. En effet, comme le rappelle Ortigues à propos des humanités, «l'esprit humain se manifeste dans ses œuvres. Les humanités littéraires qui étudient les auvres de l'esprit ont précédé historiquement la formation des sciences sociales, qui veulent être une étude plus directe des activités humaines (par voie d'observation et d'hypothèse) » :

Le latin humanitas, lorsqu'il traduit le grec païdeia, signifie «culture », " éducation », « civi-
Cette réflexion est donc propice à observer ce que la revue Sens-Dessous appelle le «dessous» qui "définit la complexité des expériences intellectuelles ou existentielles vécues, régulièrement évacuée par les discours consensuels ou la recherche d'une vérité d'un seul tenant». 
lisation ". L'humanisme est issu d'une double tradition, hellénistique (ou grécolatine) et judéo-chrétienne (hébraïque et grecque), l'humanisme a transformé l'idée même de tradition. La conception médiévale de celle-ci était d'ordre ecclésiastique ou juridico-théologique. En soumettant les auteurs sacrés aux mêmes méthodes d'exégèse que les auteurs profanes, l'humanisme a fait de la tradition des anciens « la culture » par excellence, celle qui a fondé l'unité spirituelle de l'Europe et l'a finalement ouverte à l'étude comparée de toutes les cultures. La transformation du concept de tradition ecclésiastique en celui de tradition humaniste ou culturelle est solidaire du développement de la critique philologique et historique.

Ce rappel est intéressant car il permet de concevoir les humanités comme partie intégrante des sciences de la culture telles que définies par François Rastier (2004 : en ligne):

Poursuivant un objectif de caractérisation, les sciences de la culture doivent être différentielles et comparées, car une culture ne peut être comprise que d'un point de vue cosmopolitique ou interculturel: pour chacune, c'est l'ensemble des autres cultures contemporaines et passées qui joue le rôle de corpus. En effet, une culture n'est pas une totalité : elle se forme, évolue et disparaît dans les échanges et les conflits avec les autres. Aussi, les cultures ne peuvent être décrites que différentiellement, comme les objets culturels qui les composent, au premier chef les langues et les textes.

La centralité des textes dans la prise en compte des sciences de la culture, et des humanités, en lien avec la philologie et la science des textes, et plus largement une sémiotique qui prendrait en considération l'ensemble des systèmes de signes, nous conduit à adopter une démarche réflexive sur la caractérisation même des humanités numériques: les considérer comme "croisement ", « transdisciplinaire » qui partirait des SHS, introduit un point de vue de nature à restreindre à la fois la nature même de ce domaine, et les pratiques qui s'y développent. Les humanités numériques doivent donc se concevoir de manière moins initialement centrée sur leur existence dans le champ des humanités d'un point de vue disciplinaire, et davantage selon une prise en compte nativement numérique (à la fois en fonction des objets, des problématiques, des ressources, modélisations, etc.). Il s'agit en fait de comprendre, de manière au moins réflexive, mais idéalement dès la conceptualisation même des $\mathrm{HN}$, et donc dès l'émergence de recherches, que le numérique modifie le point de vue que les humanités ont de leur domaine, agissant non comme une dimension ajoutée, une « plus-value » ou un «biais » selon la manière dont on peut qualifier l'apport, mais comme un prisme qui remodèle, récursivement, le champ des humanités. Il n'est pas question en disant cela de faire table-rase de ce que les humanités ont produit avant les $\mathrm{HN}$, mais considérer que l'existence des HN modifie l'écologie des humanités.

Cela conduit à deux distinctions à opérer:

Sur la nature du domaine, le prisme SHS (ou LSHS, si les lettres y sont intégrées) conduit à concevoir le numérique soit comme un terrain, soit comme un moyen, sans le concevoir comme un angle d'approche, en s'intéressant à ce qui sous-tend ce terrain ou ce moyen (nous y reviendrons dans la suite); 
Sur les pratiques, on se trouve donc souvent confronté au recours à des outils génériques, que l'on peut appliquer aux « humanités »; or, les sciences informatiques développent souvent, des travaux spécifiques sur des objets similaires, mais dans une perspective différente que celles des HN.

Plutôt que d'être conçues dans l'inter/trans/ pluri-discipline, les HN peuvent être conçues comme un nouveau domaine ou paradigme de recherche, qui mobilisent une appréhension scientifique distincte de celle des humanités ou de l'informatique. Du point de vue de l'outillage même, celui-ci doit être pensé spécifiquement pour répondre aux besoins en $\mathrm{HN}$, et intégrer dans sa conception même le regard que portent les humanités sur le numérique.

Aussi, cette réflexion sur les HN doit nécessairement s'accompagner, de manière aussi importante que pour les humanités, d'une définition de ce que recouvre le "numérique », et le recours plus large à l'informatique. Il faut que l'informatique rencontre les humanités, et pas seulement que les humanités utilisent l'informatique pour les besoins numériques.

\section{Informatique, numérique, digital: les don- nées en question}

Non seulement ces trois termes ne se recouvrent pas, mais en outre les enjeux de traduction laissent entrevoir des appréhensions différentes de ce domaine. Milad Doueihi (2015 : 711) décrit ainsi le passage de l'informatique au numérique:

De l'informatique (qui certes n'a pas entièrement disparu) au numérique, on passe d'une technicité, souvent exagérée et cultivée pour elle-même, mais exigeant une certaine compétence technique, à des usages plus communs, exigeant d'autres compétences : celles que valorise une nouvelle sociabilité en ligne, peuplée de textes, animée par des «partages ». Et aujourd'hui, c'est par rapport à cette pratique numérique populaire que les travaux en humanités numériques doivent être aussi pensés.

En effet, on sait par exemple que " les messages publiés sur Twitter, pourtant limités chacun à cent quarante caractères, représentent 7 To par jour,

\section{Les humanités}

numériques doivent donc se concevoir de manière moins initialement centrée sur leur existence dans le champ des humanités d'un point de vue disciplinaire, et davantage selon une prise en compte nativement numérique (à la fois en fonction des objets, des problématiques, des ressources, modélisations, etc.). 
soit une demi-BNF quotidienne » (Ganascia 2015), et que de plus en plus d'outils, projets, recherches, se concentrent sur l'analyse, l'extraction, la représentation, de données sociales numériques. De plus en plus de logiciels, parfois assez intuitifs et fonctionnels, bien documentés, enrichis de tutoriels, donnent accès à une pratique d'analyse numérique avancée. Saluons par exemple le développement du logiciel Iramuteq (que nous utilisons largement), qui est en fait une interface pour $\mathrm{R}$, et qui donne accès à des calculs statistiques, des classifications, sans avoir à entrer dans le paramétrage des fonctions ou algorithmes. Pourtant, pour avoir une vision claire des résultats produits, de la qualité des données (selon les objectifs de recherche), il est important de connaître les spécificités des différentes fonctions statistiques, probabilistes, de considérer l'impact des systèmes de représentation, de visualisation, d'exploration, des données.

Et réciproquement, certaines des tâches ou des objectifs des humanités numériques sont abordées par les sciences informatiques, en tant qu'objets de recherche, mais ne sont rarement investies dans les projets en $\mathrm{HN}$, si ce n'est sous la forme de la partie «ingénierie » des projets. Il peut y avoir un biais « utilitaire » du numérique par les humanités, qui explique par exemple qu'un des chapitres du récent ouvrage de Mounier (2018, p. 45) s'intitule: «Ce que l'ordinateur apporte aux humanités ». On comprend bien sûr que ce n'est pas l'ordinateur en tant que tel qui apporte aux humanités, mais les programmes, ressources, outils, etc., qui sont développés. Mais avec la facilité croissante de l'usage des outils informatiques, la conception même des outils tend à être mise en retrait, au profit de leur usage, simplifié. Or, derrière ces outils, il y a des recherches fondamentales, qui mobilisent des objets de recherche du point de vue informatique. Cet « oubli » des recherches fondamentales produites par les chercheurs en informatique, a été pris en compte par le Groupe de travail (GT) DAHLIA (http://dahlia.egc. asso.fr) qui « a pour but de réunir les acteurs (chercheurs ou institutions) qui s'intéressent, dans le cadre des humanités numériques, voire le patrimoine culturel, à la gestion des données mais aussi à leur analyse afin de produire des connaissances ». Il est alors important que dans une telle communauté, experte en "gestion de données », et souvent en interaction avec les spécialistes des données traitées, soit en interaction permanente avec les chercheurs en humanités, afin que les résultats ne soient pas seulement la production d'outils inductifs clef en main (comme le machine learning), mais élaborer dans la problématisation même de ses objets et méthodes, une gestion/fouille des données sémiotiques, prises en compte dans leur environnement signifiant propre.

Ce groupe, soutenu par l'Association EGC (Extraction et gestion des connaissances) part du constat que de «nombreux chercheurs de la communauté de l'analyse et la gestion de données développent des nouveaux modèles, algorithmes et logiciels permettant de traiter efficacement des données complexes ", que " ces outils innovants sont assez souvent réalisés en travaillant conjointement avec des chercheurs d'autres disciplines que l'In- 
formatique, en particulier dans les Sciences $\mathrm{Hu}-$ maines et Sociales (SHS): science de l'information et de la communication (SIC), sociologie, histoire, géographie, etc.. », mais que " malgré cet engouement, force est de constater que le courant des HN n'a été que peu mis en avant dans la communauté française en Informatique alors qu'il est très vivace dans certaines disciplines des SHS, telles que les SIC ». Il faut reconnaître que les travaux en informatique qui portent sur des humanités doivent relever le défi de considérer des données signifiantes, et non pas seulement des signifiants (mots, images, constructions): les algorithmes produits, les outils développés, doivent faire en sorte de conserver le signifié dans leur appréhension des données, pour que le sens puisse être pensé comme un objet de recherche atteignable.

Ce GT rassemble « les chercheurs en Informatique travaillant sur ce type de questions, en lien fort avec des partenaires d'autres disciplines issues des SHS ». Le changement de point de vue est intéressant, car le patrimoine, champ d'étude pilote, est considéré comme un "sous-domaine des sciences humaines et sociales ", " avec ses œuvres, documents bibliographiques et analyses/études réalisées sur les ouvres »: « dans ce contexte, les sciences et technologies peuvent venir en aide pour résoudre les problématiques du patrimoine culturel dans la gestion et l'analyse de données qui génèrent des questionnements de recherche ».

Aussi, l'informatique ne saurait se confondre avec le numérique, du point de vue des chercheurs en informatique, puisque pour les humanités numériques (voir Dacos et Mounier, 2014 : 6) le numérique peut renvoyer à trois acceptions: " Le numérique comme instrument de recherche; le numérique comme outil de communication; le numérique comme objet de recherche », ceci constituant un complexe " appelé à redéfinir l'ensemble des champs de la recherche en sciences humaines et sociales ». Selon eux, pour la situation française, il y a des acteurs des humanités numériques, «mais aucun n'est structuré en tant que « centre » au sens où nous l'entendons désormais » (ibid.: 43). Mais
Il faut reconnaître que les travaux en informatique qui portent sur des humanités doivent relever le défi de considérer des données signifiantes, et non pas seulement des signifiants (mots, images, constructions): les algorithmes produits, les outils développés, doivent faire en sorte de conserver le signifié dans leur appréhension des données, pour que le sens puisse être pensé comme un objet de recherche atteignable. 
derrière ce problème de polysémie du «numérique ", se pose davantage encore une question épistémologique de fond, que Meunier (2017) résume bien en disant qu' « il n'y a pas d'humanités dites numériques s'il n'y a pas de modélisation formelle contenant des systèmes symboliques mathématiques calculables traduisibles en algorithmes ». Pour lui, « tout projet sérieux dans les Humanités numériques, parce qu'utilisant l'ordinateur, met en place implicitement ou explicitement une modélisation formelle ». C'est cette mise en place qu'il est nécessaire de mettre en exergue, car c'est dans ces processus de modélisation formelle que se jouent les liens entre humanités et informatique, par l'intermédiaire du numérique. La définition de Dacos et Mounier montre que le numérique est un construit épistémique, un objet, et/ou un outil. Mais dans toutes ses acceptions donc, il ne saurait se confondre avec l'informatique, ou le « digital ». L'enjeu des HN est peut-être de considérer que le numérique qualifie tout autant qu'il catégorise les humanités par la manière dont il mobilise des savoirs, des méthodes, et qu'il induit une conceptualisation de son objet d'une manière différente de celle proposée par les humanités d'un côté, et de l'informatique de l'autre. Ce point est un sujet complexe intégré dans une réflexion en cours, mais il reste que cette caractérisation du numérique conduit à repenser certaines dichotomies qui caricaturent parfois le rapport du numérique aux humanités.

\section{Qualitatif/quantitatif, conceptuel/formel, sémiotique/numérique}

Sur ces questions épistémologiques, le travail mené par Jean-Guy Meunier à l'UQAM est important. Meunier 2014 retrace la généalogie de la constitution des humanités numériques, mais donne une dimension comparative en fonction des sphères d'apparition :

Dans les années 2000, en concurrence avec l'émergence des technologies informatiques, est apparu un programme de recherche original et novateur habilement appelé les Digital Humanities. Pour le monde anglophone, ce nom orientait des recherches dans une voie plus générale que ne le faisait la dénomination antérieure de Computers and the Humanities. Dans le monde francophone, l'expression anglaise, traduite par l'expression "les humanités numériques » est plus récente et n'est pas sans poser des questions, non pas relativement au qualitatif « digital », mais plutôt au substantif « humanités ». Comme on le sait ces termes humanités et les humanities ne couvrent pas les mêmes territoires disciplinaires dans les deux langues. En anglais: humanities est un terme traditionnel pour couvrir une partie de l'ensemble complémentaire des sciences dites « dures ». Il touchera surtout les sciences liées à la culture telles la sociologie, l'anthropologie culturelle, les sciences du langage, le droit, les pratiques artistiques (musique, théâtre, peinture, danse) la philosophie critique, la littérature, et même le journalisme et le cinéma. Dans une acception plus précise, les humanities couvriront les arts, les lettres, les media mais incluront plusieurs disciplines en sciences humaines critiques, par exemple la sociologie, la philosophie, etc. Dans le monde francophone, italien, espagnol, les humanités désignent généralement plus une tradition intellectuelle et même éthique de type humaniste d'inspiration érasmienne qu'un champ disciplinaire universitaire.

La dénomination «Computers and the Humanities» est intéressante, car elle inverse l'ordre dans lequel se présentent les Humanités et le Numérique/l'Informatique. Même si cela peut nous paraître un peu contre-intuitif, il est possible d'appréhender les humanités numériques par le prisme 
directement informatique, c'est-à-dire que l'appréhension des objets d'étude se fasse dans des perspectives de sciences de la donnée, d'algorithmie, de traitement de l'information. C'est ce que font nombre de chercheurs de la $27^{\mathrm{e}}$ section du CNU (Conseil National des Université, institution nationale française) par exemple, dont les objets d'étude sont directement des objets également traités par les humanités. Bien souvent, pour opposer ces approches, les débats portent sur l'analyse qualitative faite par les humanités, et quantitatives faites par l'informatique.

Or, la séparation qualité/quantité ne recouvre pas la distinction entre humanités et informatique. Il existe, dans ces domaines, des traditions intellectuelles, des courants, des écoles, des approches, qui sont notamment fondées sur des conceptions spécifiques des connaissances, de la science, de la représentation des savoirs. Tout cela permet ainsi d'aborder les différents modèles qui peuvent présider aux travaux scientifiques. Ceci est décrit par Meunier (2018), et amène à des considérations qui changent notre rapport à l'informatique. En effet, si l'informatique, tel qu'elle est appréhendée par les humanités numériques, se base sur un modèle computationnel, ce modèle computationnel se fonde sur un modèle formel, et le choix d'un modèle formel spécifique n'est pas neutre:

un modèle formel ne porte pas nécessairement sur du quantitatif. Aussi, on trouvera des formalismes logiques, géométriques, topologiques, grammaticaux, etc. Certains utiliseront des symboles iconiques (graphes, images, etc.). Dans tous ces formalismes, on pourra trouver divers types de symboles: tels des constantes, des variables, des opérateurs, etc.

Ce qui peut donc distinguer une approche informatique des humanités numériques d'une approche qui donne la priorité aux humanités, ce ne serait pas forcément l'intérêt premier pour l'ordinateur, mais plutôt le choix d'un modèle formel adéquat et permettant la computation. Pour que les humanités numériques existent et proposent une cohérence, il faut donc que les données signifiantes soient prises en compte dans la perspective du modèle formel choisi, qui permettra leur com-
Ce point est un sujet complexe intégré dans une réflexion en cours, mais il reste que cette

caractérisation du numérique conduit à repenser certaines dichotomies qui caricaturent parfois le rapport du numérique aux humanités. 
putation par un ordinateur. De fait, « ces modèles formels mathématiques sont omniprésents tant dans les sciences naturelles que dans les sciences humaines et sociales ». Ainsi, lorsque Meunier s’intéresse à la difficulté de la notion de computationnalité pour la sémiotique, il précise qu' "une théorie sémiotique de type computationnel ne peut exister que si elle en appelle à de la modélisation formelle de type mathématique (non pas nécessairement quantitatif), dont les énoncés, formules ou équations permettent la calculabilité. Ce n'est qu'à cette condition qu'une sémiotique peut être computationnelle ».

La modification réciproque des rapports entre humanités et informatique est donc profonde, et recouvre des enjeux plus profonds qu'il n'y paraît. Ainsi Jean-Guy Meunier (2019) précise que «certains experts des humanités croient, cependant, que cette technologie informatique ne fait que donner de nouveaux types d'accès au domaine des humanités et que leur analyse n'en continue pas moins à être de type interprétatif ». Le travail des humanités peut alors consister à commenter les résultats produits grâce à un ordinateur et "l'informatique apparaît simplement comme un nouvel outil d'assistance dans l'approche des objets sémiotiques par la numérisation, l'archivage, la fouille, etc. ». Ceci reviendrait à penser que «l'informatique n'affecterait pas sérieusement l'intégrité des pratiques classiques des humanités ». Or, le grand intérêt épistémologique du travail de Meunier est de replacer l'articulation entre humanités et informatique à un autre niveau. Ce déplacement converge vers la réorganisation des champs/disciplines que nous avons proposée précédemment, en confrontant humanités et informatique/numérique. En effet, si ces approches de l'informatique «que l'on comprend comme étant surtout quantitatives ne sont alors qu'une redistribution des cartes des sciences humaines et sociales ", entérinant une nette opposition entre l'informatique et les humanités ${ }^{2}$, nous pensons qu'il est possible de penser les choses en termes de co-construction, au niveau de la conception même des objets et problématiques de recherche.

\section{Dépasser la contradiction entre humanités et numérique}

Dans une conférence intitulée « Humanités Numériques Ingénierie du sens ou herméneutique matérielle? » donnée à l'université de Cergy-Pontoise, Jean-Guy Meunier note "une tension, un paradoxe, une contradiction entre Humanités et numérique », et se pose les questions suivantes: Comment penser cette relation? Quelles formes de connaissances sont les HN ? Peut-on les voir comme un mode de connaissance scientifique?

Un détour par les questions de disciplinarité (inter/poly/pluri/transdisciplinarité), telles que proposées par Morin (1994) aide à mieux situer les choses:

En fait, ce sont des complexes d'inter, de poly, et de transdisciplinarité qui ont opéré et qui ont joué un rôle fécond dans l'histoire des sciences; il faut retenir les notions clés qui y sont impliquées, c'est-à-dire coopération, et mieux, articulation, 
objet commun et mieux, projet commun. Enfin, ce n'est pas seulement l'idée d'inter- et de transdisciplinarité qui est importante. Nous devons «écologiser » les disciplines, c'est-à-dire tenir compte de tout ce qui est contextuel y compris des conditions culturelle et sociales, c'est-à-dire voir dans quel milieu elles naissent, posent des problèmes, se sclérosent, se métamorphosent. Il faut aussi du métadisciplinaire, le terme « meta » signifiant dépasser et conserver. On ne peut pas briser ce qui a été créé par les disciplines; on ne peut pas briser toute clôture, il en est du problème de la discipline, du problème de la science comme du problème de la vie: il faut qu'une discipline soit à la fois ouverte et fermée. En conclusion, à quoi serviraient tous les savoirs parcellaires sinon à être confrontés pour former une configuration répondant à nos attentes, à nos besoins et à nos interrogations cognitives.

On voit donc que les humanités numériques sont un domaine propice à faire avancer l'histoire des idées et l'histoire des sciences, puisqu'elles nécessitent une approche métadisciplinaire qui donne à voir les enjeux de chaque domaine (humanités et informatique) et les particularités des disciplines spécifiques (littérature, histoire, linguistique, etc.; fouille de données, traitement de l'image, gestion des connaissances, etc.).

Pour prendre « de la hauteur » vis-à-vis de ce questionnement, et adopter une position réflexive, nous pouvons interroger la « dynamique cyclique » proposée par Jean-Guy Meunier, avec la représentation des relations réciproques entre les modèles conceptuels, formels mathématiques, formels computationnels, et physiques informatiques:

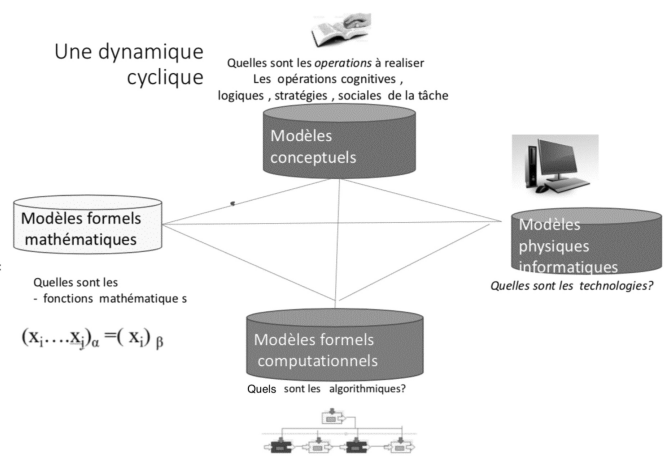

Dynamique des modèles (Meunier 2019b)
La modification réciproque des rapports entre humanités et informatique est donc profonde, et recouvre des enjeux plus profonds qu'il n'y paraît.
2. À propos de la rencontre entre la sémiotique et l'informatique, Meunier rappelle que "plusieurs formulations souvent synthétiques, mais quelques fois simplificatrices ou inadéquates exprimeront cette rencontre du sémiotique et de l'informatique en tant qu'opposition entre : le quantitatif et le qualitatif, le descriptif et l'interprétatif, l'expérimentation et l'interprétation, les sciences naturelles et les sciences humaines, les Naturwissenchaften et les Geistwissenshaften, etc. ». 
Cette articulation permet de rebattre les cartes de la séparation humanités/informatique, puisque si le «numérique » peut s'incarner dans les technologies utilisées et mobilisées, les autres modèles transcendent les séparations établies entre humanités et informatique, et il s'agit finalement de pouvoir élaborer des modélisations cohérentes, entre conceptualisation, formalisation mathématique, conception d'algorithmes.

\section{Conclusion}

Les humanités numériques donnent lieu à de nombreux travaux, projet, et s'incarnent diversement dans des pratiques, et des traditions parfois éloignées. Si les recherches en HN ont en commun de présenter le recours au numérique pour renouveler les analyses traditionnelles, il y a bien souvent une séparation entre humanités et numérique, qui crée des tensions, voire des incompréhensions, entre acteurs. Dans cet article, nous avons essayé de montrer que pour aller plus loin dans leurs objectifs, les HN doivent pouvoir créer un lien réciproque entre humanités et numérique, par la coconstruction des objets, problèmes, méthodes, et traitements. La perspective adoptée par Meunier permet de sortir des considérations clivantes entre qualité et quantité, pour redistribuer les perspectives des deux domaines selon les différents types de conceptualisation. Si humanités et informatique co-construisent la conceptualisation et la formalisation, mathématique et informatique, des objets signifiants qu'elles veulent traiter, alors les HN sortiront d'un rapport de complémentarité entre, par exemple, le texte et l'ordinateur, pour appréhender conjointement un objet que l'on saisit par sa conceptualisation et sa représentation potentiellement computable. Bien sûr, les humanités garderont leur capacité à interpréter, et l'informatique à traiter de manière puissante, mais l'un et l'autre seront en mesure d'appréhender leur objet comme un tout cohérent.

Julien Longhi

\section{Bibliographie}

- Dacos M., Mounier P., 2014, Humanités numériques. État des lieux et positionnement de la recherche française dans le contexte international, Paris, Institut français/ministère des Affaires étrangères pour l'action culturelle. Accès : http://www.institutfrancais.com/sites/default/files/if_humanites-numeriques.pdf.

- Doueihi M., 2015, «Quelles humanités numériques? ”, Critique, 819-820, P.. 704-711.

- Ganascia J.-G., 2015, « Les big data dans les humanités », Critique, 819820, P. 627-636.

- Longhi J. (dir.), 2017, Humanités numériques, corpus et sens, Questions de communication, 2017/1 ( $\left.\mathrm{n}^{\circ} 31\right)$. 
- Morin E., 1994, "Sur l'interdisciplinarité ». Accès: http://ciret-transdisciplinarity.org/bulletin/ b2c2.php.

- Mounier P., 2018, Les humanités numériques.

Une histoire critique, Paris, Éditions de la Maison des sciences de l'homme

- Meunier J.-G., 2014, « Humanités numériques ou computationnelles: Enjeux herméneutiques », Sens public. Accès: http://www.sens-public.org/ spip. php?article1121

- Meunier J.-G., 2017, « Humanités numériques et modélisation scientifique ", Questions de communication, 2017/1 ( $\left.\mathrm{n}^{\circ} 31\right)$, 19-48. URL: https:// www.cairn.info/revue-questions-de-communication-2017-1-page-19.htm.

- Meunier J.-G., 2018, «Vers une sémiotique computationnelle? ", Applied semiotics, 26.

- Meunier J.-G., 2019, « Humanités Numériques: Ingénierie du sens ou herméneutique matérielle? », Conférence donnée à l'Institut d'études avancées, 9 avril 2019, Université de Cergy-Pontoise.

- Meunier J.-G., 2019 (à paraître), « La rencontre du sémiotique et du "numérique »: Le rôle d'une modélisation conceptuelle », Semiotica.

- Ortigues E, « Sciences Humaines », Encyclopedia Universalis [en ligne], consulté le 31 mars 2019. URL: http://www.universalis-edu.com/encyclopedie/sciences-humaines/

- Rastier F., 2004, « Doxa et lexique en corpus pour une sémantique des idéologies », Texto! Accès: http://www.revue-texto.net/Inedits/Rastier/Rastier_Doxa.html
On voit donc que les humanités numériques sont un domaine propice à faire avancer l'histoire des idées et l'histoire des sciences, puisqu'elles nécessitent une approche métadisciplinaire qui donne à voir les enjeux de chaque domaine (humanités et informatique) et les particularités des disciplines spécifiques (littérature, histoire, linguistique, etc.; fouille de données, traitement de l'image, gestion des connaissances, etc.). 
